illness. Abnormality as a criterion is not sufficient to distinguish illness from other forms of deviance. The meaning of the terms illness and disease is derived from the context of physical, bodily conditions and implies more than deviation from the norm. These concepts characterise processes which are discrete from the subject's will or intentionality and whose course is biologically determined. To suggest that behaviours and utterances are "symptoms" of illness is therefore to classify them as events that have no relation to the voluntary activity and purposes of the individual and are therefore devoid of meaning. This seems to me to be an impoverished approach to the myriad of complex human behaviours that comprise psychiatric problems and is likely to hamper the process of finding imaginative solutions.

Characterising mental disorders as existential conditions rather than as illnesses does not mean that medical techniques have no place in helping people to manage or survive them. A different emphasis in psychiatry might liberate psychiatrists and patients alike from the shackles of the "illness" paradigm.

KERR, A. \& Howarth, P. (1996) Commentary on " 'Audible thoughts' and 'speech defect' in schizophrenia". British Journal of Psychiatry, 168, 538-539.

Szasz, T. (1996) 'Audible thoughts' and 'speech defect' in schizophrenia. A note on reading and translating Bleuler. British Journal of Psychiatry, 168, 533-535.

\section{J. MONCRIEFF}

Institute of Psychiatry

London SE5 8AF

SIR: Szasz (1996) has provided us with another sample of his inimitable linguistic legerdemain, this time concerning the phenomenology of schizophrenic thought disorder and auditory hallucinations.

Firstly, contra Szasz, there is a clear difference between hearing one's own thoughts spoken aloud, as if they were coming from outside oneself, by a (stranger's) voice at the same time as one is thinking them (Gedankenlautwerden) and "hearing" one's own inner voice or even thinking aloud. There is no confusion between these two distinct phenomena in psychopathology.

The comparison between schizophrenic thought insertion and "projection" is a typical Szaszian disanalogy. The belief that others are somehow beaming their thoughts into one's head is categorically different from accusing others of having feelings that one is unwilling to recognise in oneself. Again the psychopathologies are unmistakably distinct, and certainly not applied post hoc after deciding whether the patient is sane or insane.

Finally, how can he assert that "ordinary medical maladies are not diagnosed by making inferences from the way the patient speaks"? We routinely do so in terms of both the form and the content of the patient's speech; the former is utilised to diagnose cerebellar staccato speech and Parkinsonian speech; the latter whenever we reach any diagnosis from the history alone; perhaps a mixture of the two with the aphasias. These 'speech defects' certainly do not arise from "incorrect use of the muscles of [the] mouth and tongue" as Szasz would have it, but from brain diseases. It also seems particularly wilful to suggest that schizophrenic speech is "deviant" in the way that a thick Yorkshire accent deviates from Received Pronunciation.

Perhaps the only worthwhile point I could draw from this paper was that we should more properly speak of schizophrenic speech disorder and inferred thought disorder since we do not have direct access to the thoughts of others.

Szasz, T. (1996) 'Audible thoughts' and 'speech defect' in schizophrenia. A note on reading and translating Bleuler. British Journal of Psychiatry, 168, 533-535.

S. WIISON

Friarage Hospital

Northallerton

North Yorkshire DL6 $1 \mathrm{JG}$

\section{Tricyclics and SSRIs}

SR: Taylor \& Lader (1996) usefully point out the potential dangers of combining tricyclic antidepressants with selective serotonin reuptake inhibitors. The tertiary referrals received at this unit, which specialises in treatment-resistant depression, indicate that this practice is becoming increasingly common and usually occurs without the monitoring of serum tricyclic antidepressant concentrations. We would like to extend their clinical recommendations by suggesting that this combination should not be routinely used. It is difficult to justify theoretically as adequate doses of tertiary amine tricyclic antidepressants would produce the same effect on cerebral amines as combinations of tricyclics and selective serotonin reuptake inhibitors. If adequate doses of tricyclic antidepressants are not tolerated then perhaps venlafaxine would be the next logical step because of its effect on both serotonin and noradrenaline neurotransmitters.

A clinically important cytochrome-mediated interaction that Taylor \& Lader do not mention is 
the induction of p450 enzymes by carbamazepine. This leads to a lowering of serum tricyclic concentrations when tricyclics are given with carbamazepine, thus reducing their antidepressant action (Leinonen et al, 1991; Preskorn, 1993). Such interactions altering the serum concentrations of tricyclic antidepressants are unlikely to be adequately appreciated until the measurement of serum tricyclic antidepressant concentrations becomes routine clinical practice, especially for cases that are difficult to treat.

Leinonen, S. A., Limsunde, P., Laukranen, V., et al (1991) Effects of carbamazapine on serum antidepressant concentrations in psychiatric patients. Journal of Clinical Pharmacology, 11, 313-318.

Preskorn, S. H. (1993) Pharmacokinetics of antidepressants: why and how they are relevant to treatment. Journal of Clinical Psychiatry, 54 (Suppl. 9), 14-34.

TAYLOR, D. \& LADER, M. (1996) Cytochromes and psychotropic drug interactions. British Journal of Psychiatry, 168, 529-532.

Geoffrey Knight National Unit for

R. J. BowSKILL P. K. BRIDGES

Affective Disorders

Maudsley Hospital

London SE5 8AZ

\section{Cross-cultural studies}

SIR: The success of the Bradford Somatic Inventory (BSI) in identifying minor psychiatric morbidity in a rural Pakistani district underlines the value of instruments which have been validated in ways that are language and culture sensitive (Mumford et al, 1996). The identification of these psychiatric cases however recalls the argument of the legitimacy of imposing Western psychiatric diagnoses in non-Western societies (Kleinman, 1977). In stating that to the casual Western visitor life in this remote district appears idyllic and contented suggests that the authors, while good intentioned, may have fallen into the trap of the ethnic psychiatrists of the past, who sought out psychopathology in exotic places while reinforcing colonial and neocolonial attitudes and behaviour (Bhugra, 1993). It is important to recognise that translation (as in establishing a diagnostic index in one setting and then translating it so that it can be used in another) does not ensure congruity of sociocultural perspectives. Translation only ensures that there is an approximate equivalence between categories whose origins lie in very different social contexts (Skultans, 1993).

It is therefore unfortunate that there is no account of how the villagers themselves interpret and articulate their distress as psychiatry itself is a Western construct and imposes its own sociocultural parameters when observations are made on its behalf. In addition, the lack of information about physical illness is a hindrance to the accurate interpretation of the findings since the correlation of deprivation with both physical and psychiatric morbidity is well recognised and the diagnosis of any psychiatric illness must be related to the presence of physical illness.

There is undoubted value in cross-cultural psychiatric research as mass migration is creating increasingly multicultural Western societies. However, when psychiatry co-opts non-Western experience in ways that would allow the distress that is derived from that experience to be interpreted and categorised in Western terms, the native interpretations are often undermined. What is also lost is the capacity to incorporate this perspective into Western formulations so that the delivery of care in multicultural Western societies can be more relevant and effective. A goal that continues to be elusive.

BhugrA, D. (1993) Cross-cultural psychiatry. Why? Where? How? International Review of Psychiatry, 5, 123-124.

KLetnman, A. (1977) Depression, somatization and the 'new cross-cultural psychiatry'. Social Science and Medicine, 11, 3-10. MuMforD, D. M., NAZIR, M., JiLANI, F., et al (1996) Stress and psychiatric disorder in the Hindu Kush. A community survey of mountain villages in Chitral, Pakistan. British Journal of Psychiatry, 168, 299-307.

Sxultans, V. (1993) The case of cross-cultural psychiatry: squaring the circle? International Review of Psychiatry, 5, 125-128.

Maudsley Hospital

G. HutCHINSON

\section{London SE5 8AZ}

\section{Lithium and weight gain}

SIR: Previous studies show that weight increases during the first two years of lithium therapy and then stabilises, that a quarter of patients do not put on weight at any stage (Vestergaard et al, 1990) and that a quarter become obese (Chen \& Silverston, 1990). Consequently the BNF mentions weight gain as a problem. This was investigated in a lithium clinic where in 17 years no patient had dropped out because of weight gain.

Forty-two unipolar and bipolar patients on lithium for 1-25 years with or without concomitant antidepressants or neuroleptics were weighed in shoeless light attire and their height measured. Using the same balance arm weighing machine and measure, controls similarly attired from the same locality and not suffering from any known 